\title{
TITLE:
}

\section{Intense blue emission from tantalum-doped silicate glass}

$\operatorname{AUTHOR}(S)$ :

Meng, XG; Murai, S; Fujita, K; Tanaka, K

\section{CITATION:}

Meng, XG ...[et al]. Intense blue emission from tantalum-doped silicate glass. APPLIED PHYSICS LETTERS 2006, 89(6): 061914.

\section{ISSUE DATE:}

2006-08-07

URL:

http://hdl.handle.net/2433/39685

\section{RIGHT:}

Copyright 2006 American Institute of Physics. This article may be downloaded for personal use only. Any other use requires prior permission of the author and the American Institute of Physics. 


\title{
Intense blue emission from tantalum-doped silicate glass
}

\author{
Xiangeng Meng, Shunsuke Murai, Koji Fujita, and Katsuhisa Tanaka ${ }^{a}$ \\ Department of Material Chemistry, Graduate School of Engineering, Kyoto University, \\ Katsura, Nishikyo-ku, Kyoto 615-8510, Japan
}

(Received 24 March 2006; accepted 20 June 2006; published online 9 August 2006)

\begin{abstract}
The authors report on an intense blue emission centered at $420 \mathrm{~nm}$ under ultraviolet excitation from pentavalent tantalum ion-doped silicate glasses. The blue emission is distinctly different from emission due to defects in silica glass, which is also reflected in both absorption spectrum and remarkably enhanced emission. The authors suggest that localized Ta $5 d^{0}$ energy level is responsible for the blue emission. (C) 2006 American Institute of Physics. [DOI: 10.1063/1.2335394]
\end{abstract}

Continuous efforts have been made to achieve effective blue emission, with an aim of realizing high power all solidstate blue lasers and full-color displays. ${ }^{1}$ Semiconductor materials (e.g., GaNs and $\mathrm{ZnO}$ ) have demonstrated their applications in an extended region due to particular advantages, such as wide direct band gap, commercial availability with large area, and high quality single crystals. Compared with bulk crystalline hosts, glass materials possess specific advantages, e.g., easy fabrication, low cost, high mechanical strength, and high chemical durability. Therefore, glasses play an indispensable role in a field of luminescent materials working in a wide range of wavelength, including ultraviolet, visible, and infrared regions. Usually, the emission in glass hosts is realized by incorporating rare earth (RE) ions or transition metal (TM) ions. The precondition is that REs and TMs possess electrons that incompletely fill $d$ or $f$ orbitals to induce electronic transitions, e.g., $f-f / d$ transitions for REs and $d-d$ transitions for TMs.

Among the group $\mathrm{V}$ elements in the Periodic Table, much attention has been paid to vanadium ions because of their promising applications in telecommunication systems. ${ }^{2-4}$ Niobium complex $\mathrm{NbO}_{4}^{3-}$ has also been investigated as a possible blue phosphor. ${ }^{5}$ In contrast, tantalum ions, usually existing as $\mathrm{Ta}^{5+}$, have not received much attention, because its completely occupied outermost electronic shell is usually regarded as a disability in electronic transition. However, it is not always the case. Here we report on an intense blue emission from pentavalent tantalum ion-doped silicate glass. It is suggested that the localized Ta $5 d^{0}$ energy level should be responsible for the blue emission.

The glass was prepared by using the conventional meltquenching method from reagent $\mathrm{SiO}_{2}, \mathrm{CaCO}_{3}, \mathrm{Na}_{2} \mathrm{CO}_{3}$, and $\mathrm{Ta}_{2} \mathrm{O}_{5}$ as starting materials. $10 \mathrm{CaO}-20 \mathrm{Na}_{2} \mathrm{O}-70 \mathrm{SiO}_{2}$ composition (in mol \%) was chosen as a glass matrix and $1 \mathrm{~mol} \% \mathrm{Ta}_{2} \mathrm{O}_{5}$ was added by replacing $\mathrm{SiO}_{2}$. In order to reduce the effect of hydroxyl ions on the luminescence, the initial powders were firstly calcined at $500{ }^{\circ} \mathrm{C}$ for $10 \mathrm{~h}$ in an electronic furnace and then ball milled in an agate container for $10 \mathrm{~h}$. The final homogenous mixture was melted at $1550{ }^{\circ} \mathrm{C}$ for $1-4 \mathrm{~h}$ in a platinum crucible. The glass melt was poured onto a stainless steel plate and then pressed with another plate to obtain clear glass. The as-manufactured glass samples, which were colorless and bubble-free, were cut into a size of $5 \times 5 \times 1 \mathrm{~mm}^{3}$ and polished for optical

${ }^{a)}$ Electronic mail: tanaka@dipole7.kuic.kyoto-u.ac.jp measurements. For comparison of emission intensity, cerium-doped zinc borosilicate glass with a composition of $19 \mathrm{SiO}_{2}-20 \mathrm{~B}_{2} \mathrm{O}_{3}-60 \mathrm{ZnO}-1 \mathrm{CeO}_{2}$ (in $\mathrm{mol} \%$ ) was also prepared through the above procedures. Optical absorption spectrum was measured with a Jasco-V570 UV-vis-NIR spectrophotometer. Measurements of excitation and emission spectra were performed with a Hitachi 850 fluorescence spectrophotometer. Electron spin resonance (ESR) measurements were carried out by using a JES-TE100 ESR spectrometer operating at $X$-band microwave frequency to detect paramagnetic defects and impurities in the glasses.

Figure 1 shows the room temperature absorption spectra of $\quad 10 \mathrm{CaO}-20 \mathrm{Na}_{2} \mathrm{O}-70 \mathrm{SiO}_{2} \quad$ (SSC) and $10 \mathrm{CaO}-20 \mathrm{Na}_{2} \mathrm{O}-69 \mathrm{SiO}_{2}-1 \mathrm{Ta}_{2} \mathrm{O}_{5}$ (SSCT). Compared with SSC, SSCT obviously possesses a broader and flat absorption band. The UV absorption of SSC is located at $205 \mathrm{~nm}$, while that of SSCT extends to $236 \mathrm{~nm}$. It should be also noticed that the maximum absorption coefficient of SSC is about $27 \mathrm{~cm}^{-1}$, while that of SSCT increases to $32 \mathrm{~cm}^{-1}$. The charge transfer between $\mathrm{O}^{2-}$ and $\mathrm{Ta}^{5+}$ may be responsible for the absorption band of SSCT.

Figure 2 presents the excitation and emission spectra of SSC (a) and SSCT (b). The excitation spectrum of SSCT is characterized as a narrow band with full width at half maximum (FWHM) of $37 \mathrm{~nm}$, while the emission a broad one with FWHM of $115 \mathrm{~nm}$. A very weak emission band at $400 \mathrm{~nm}$ occurs for SSC when excited with $210 \mathrm{~nm}$ light, which may be imposed to impurity in raw materials (e.g., $\left.\mathrm{Fe}^{3+}\right){ }^{6}$ Relative to the maximum of the emission observed for SSC, the emission intensity of SSCT is $\sim 100$ times

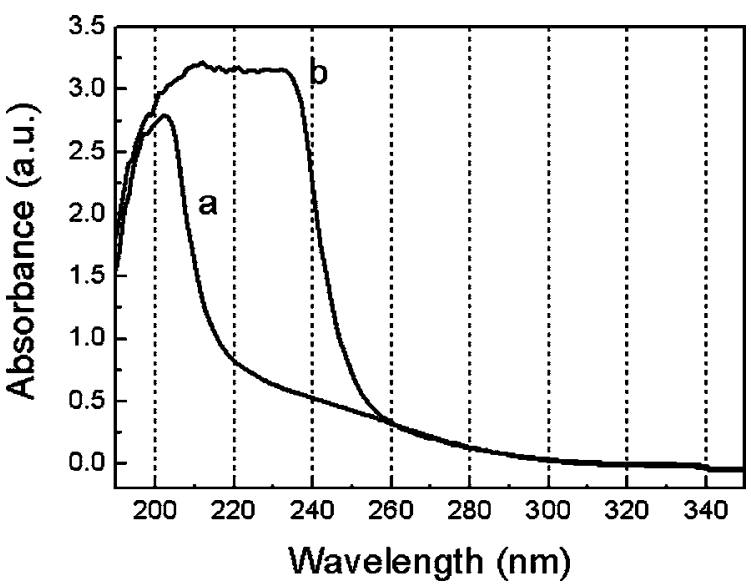

FIG. 1. Optical absorption spectra of SSC (a) and SSCT (b). 


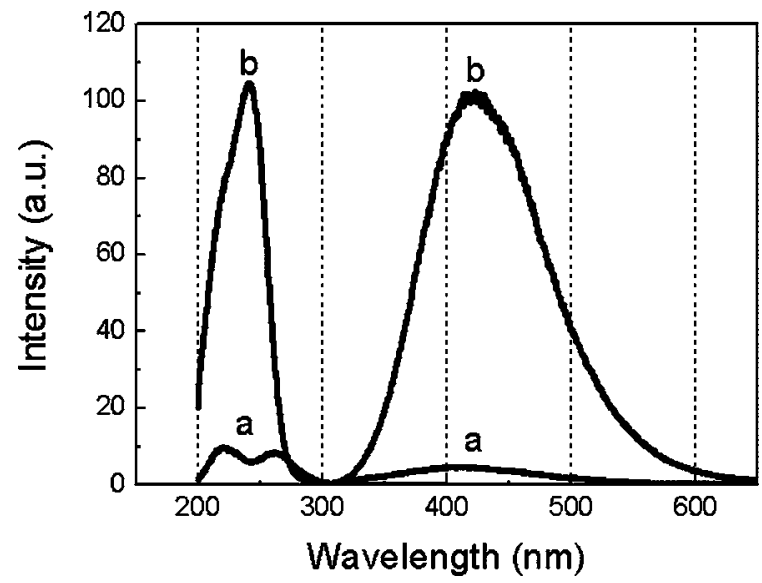

FIG. 2. Excitation (left) and emission (right) spectra of SSC (a) (monitored at $400 \mathrm{~nm}$ and excited at $210 \mathrm{~nm}$ ), respectively and SSCT (b) (monitored at $420 \mathrm{~nm}$ and excited at $245 \mathrm{~nm}$ ), respectively.

larger when excited at $254 \mathrm{~nm}$, which is the working wavelength of commercial mercury UV lamps. When the samples were exposed to $254 \mathrm{~nm}$ UV lamp irradiation, the emission from SSC became invisible while tantalum ion-doped glass showed blue intense emission. The emission intensity for SSCT is approximately ten times higher than that for ceriumdoped zinc borosilicate glass, the latter exhibiting strong blue emission under UV excitation. ${ }^{7}$

One possible origin of the blue emission from SSCT is defects induced by incorporation of tantalum ion. It is well known that there are various defects in silica glass, e.g., nonbridging oxygen hole center ( $\equiv \mathrm{Si}-\mathrm{O} \uparrow, \uparrow$ means one unpaired electron.), ${ }^{8}$ peroxy radicals ( $\left.\equiv \mathrm{Si}-\mathrm{O}-\mathrm{O} \uparrow\right),{ }^{9} E^{\prime}$ ( $\equiv \mathrm{Si} \uparrow),{ }^{10}$ and $B_{2}$ (三Si-Si $\left.\equiv\right) .{ }^{11}$ The ESR spectra of SSC and SSCT are shown in Fig. 3, in which three weak signals appear. The $g_{1}=4.2636$ signal should be ascribed to $\mathrm{Fe}^{3+}$ impurity $^{12}$ responsible for the $400 \mathrm{~nm}$ emission in SSC, while the other two may be associated with paramagnetic defects in silicate glasses ${ }^{13-15}$ or impurities (e.g., $\mathrm{Cu}^{2+}, \mathrm{Fe}^{3+}$ ) (Ref. 16) in raw materials because similar signals are also observed in sodium carbonate used as the raw material in the present work. These paramagnetic defects present in silica glass have not been reported to emit photons at blue wavelength region, while the $B_{2}$ defect, a kind of diamagnetic one, can yield emission at $3.16 \mathrm{eV}(392 \mathrm{~nm})$ under UV irradiation. In that case, a strong emission band at $4.42 \mathrm{eV}$

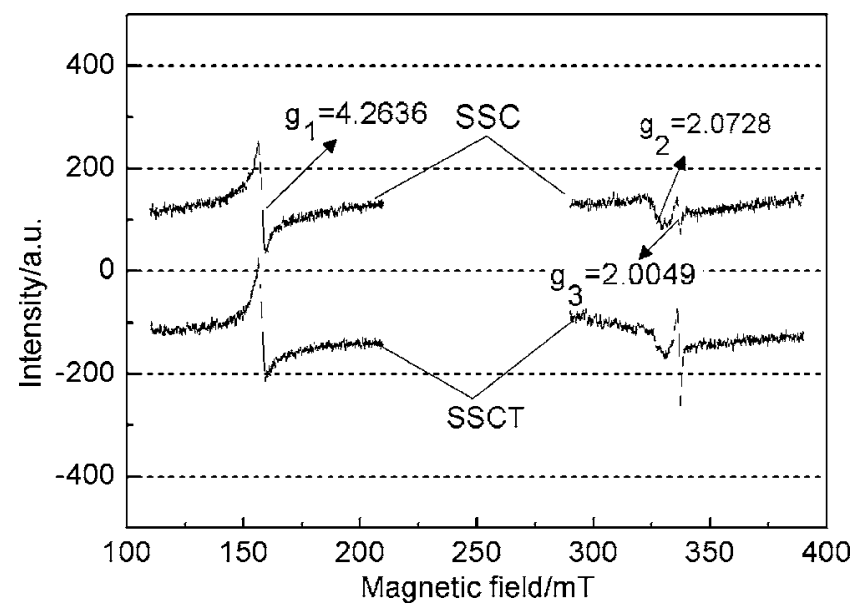
FIG. 3. Electron spin resonance spectra of SSC and SSCT.
Downloaded 31 May 2007 to 130.54 .110 .22 . Redistribution subject to AIP license or copyright, see http://apl.aip.org/apl/copyright.jsp

$(280 \mathrm{~nm})$ as well as a very weak emission at $2.74 \mathrm{eV}$ $(452 \mathrm{~nm})$ is usually accompanied. ${ }^{11}$ However, such emission bands were not observed for the present SSCT glass. In addition, the $B_{2}$ defect, reflected in the absorption spectrum, possesses an absorption band at $5.0 \mathrm{eV}$ with absorption coefficient less than $1 \mathrm{~cm}^{-1} \cdot{ }^{11}$ As described above, incorporation of tantalum ions resulted in a broader absorption band with absorption coefficient as large as $32 \mathrm{~cm}^{-1}$, which indicates that the possible $B_{2}$ defect should have a negligible effect on the emission of SSCT.

Based on the above analyses, we intend to ascribe the remarkably enhanced blue emission to electronic transitions related to tantalum ions. Usually, the tantalum ions are present as a pentavalent state with the corresponding electronic configurations as $5 d^{0}$. Therefore, the $d$ - $d$ transition does not take place. The $420 \mathrm{~nm}$ blue emission from tantalum ions is rarely known up to now. It was reported that $\mathrm{Ta}_{2} \mathrm{Zn}_{3} \mathrm{O}_{8}$ thin film emitted photons at $385 \mathrm{~nm}$ when excited by $236 \mathrm{~nm}$ UV light and $420 \mathrm{~nm}$ blue emission could be observed by filtering. ${ }^{17}$ In that case, the excitation spectrum was also characterized as a narrow band, while the emission spectrum exhibited a broad one. The spectral properties are similar to those of the present work. A metal-to-ligand radiative transition mechanism was proposed to interpret this unusual emission. ${ }^{17}$ The excited state is the $5 d^{0}$ state of the tantalum ions whereas the ground state is the $2 p^{6}$ state associated with the oxide ions. Octahedrally coordinated $\left(\mathrm{TaO}_{6}\right)^{7-}$ and other octahedrally coordinated transition metal ions (e.g., $\mathrm{MoO}_{6}, \mathrm{WO}_{6}$, and $\mathrm{TiO}_{6}$ ) (Ref. 18) are known as rather efficiently luminescent centers via this metal-to-ligand transition. Furthermore, it is known that the $\mathrm{NbO}_{4}^{3-}$ complex is a kind of self-luminescent center, in which blue emission can be ascribed to the transition between $\mathrm{Nb} 4 d$ and $\mathrm{O} 2 p$ orbitals. ${ }^{5}$ Given the similarities in spectral properties between $\mathrm{Ta}_{2} \mathrm{Zn}_{3} \mathrm{O}_{8}$ and SSCT, we propose the following mechanism, as shown in Fig. 4. An electron in $\mathrm{O} 2 p$ orbital is excited to Ta $5 d^{0}$ by UV photon and then thermally relaxed to the lowest vibrational state of Ta $5 d^{0}$ energy level. Finally, the excited electron relaxes to the valence band accompanied by emission of a blue wavelength photon.

In summary, we have realized an intense blue emission from tantalum ion-doped silicate glass. The localized energy level of tantalum ion is proposed to be responsible for the emission. The tantalum-doped glasses possess some specific advantages. Firstly, the glass investigated herein, a traditional soda lime silicate glass with excellent thermal stability, mechanical properties, and chemical durability, has been extensively utilized for commercial purposes in many fields. Secondly, valence control, which is necessary for other blue emitting activators, such as $\mathrm{Eu}^{2+}, \mathrm{Sn}^{2+}$, or $\mathrm{Cu}^{+}$, is not required for tantalum-doped blue emitting glasses. In particu-

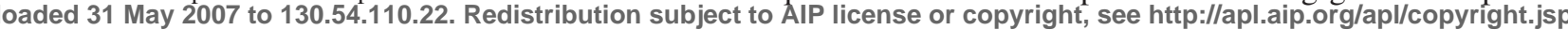


lar, it is of interest that the blue emission can also be realized by incorporating tantalum ions into nonsilicate glass hosts, ${ }^{19}$ which indicates that tantalum ions will open up a new frontier in luminescent materials as a novel activator. Although complete understanding of the mechanism requires further investigation, the easy fabrication method and high emission intensity, together with the above-mentioned advantages for SSCT provide a promising material in display and blue laser fields.

This study was partially supported by a Gr;ant-in-Aid for Scientific Research (No. 18360316) from the Ministry of Education, Culture, Sports, Science, and Technology, Japan.

${ }^{1}$ R. Gvishi, G. S. He, P. N. Prasad, U. Narang, M. Li, F. V. Bright, B. A. Reinhardt, J. C. Bhatt, and A. G. Dillard, Appl. Spectrosc. 49, 834 (1995).

${ }^{2}$ R. B. Bylsma, P. M. Bridenbaugh, D. H. Olson, and A. M. Glass, Appl. Phys. Lett. 51, 889 (1987).

${ }^{3}$ A. Partovi, J. Millerd, E. M. Garmire, M. Ziari, W. H. Steier, S. B. Trivedi, and M. B. Klein, Appl. Phys. Lett. 57, 846 (1990).

${ }^{4}$ M. Ziari, W. H. Steier, P. M. Ramon, M. B. Klein, and S. Trivedi, J. Opt. Soc. Am. B 91, 461 (1992).
${ }^{5}$ S. H. Shin, D. Y. Jeon, and K. S. Suh, J. Appl. Phys. 90, 5986 (2001).

${ }^{6}$ B. Schmitz and G. Lehmann, Chem. Phys. Lett. 63, 438 (1979).

${ }^{7}$ K. Annapuma, R. N. Dwivedi, P. Kundu, and S. Buddhudu, Mater. Lett. 58, 787 (2004).

${ }^{8}$ M. Stapelbroek, D. L. Griscom, E. J. Friebele, and G. H. Sigel, Jr., J. Non-Cryst. Solids 32, 313 (1979).

${ }^{9}$ D. L. Griscom, J. Ceram. Soc. Jpn. 99, 923 (1991).

${ }^{10}$ J. R. Chavez, S. P. Karna, K. Vanheusden, C. P. Brothers, R. D. Pugh, B. K. Singaraju, W. L. Warren, and R. A. B. Devine, IEEE Trans. Nucl. Sci. 44, 1799 (1997).

${ }^{11}$ L. Skuja, J. Non-Cryst. Solids 239, 16 (1998).

${ }^{12}$ K. Tanaka, Ph.D. thesis, Kyoto University, 1990.

${ }^{13}$ Y. Wu and A. Stesmans, Phys. Rev. B 38, 2779 (1988).

${ }^{14}$ K. Moritani, I. Takagi, and H. Moriyama, J. Nucl. Mater. 329-333, 988 (2004).

${ }^{15}$ K. Kajihara, L. Skuja, M. Hirano, and H. Hosono, J. Non-Cryst. Solids 345-346, 219 (2004).

${ }^{16}$ R. C. Nicklin, H. A. Farach, and C. P. Poole, Jr., J. Chem. Phys. 65, 2998 (1976).

${ }^{17}$ P. D. Rack, M. D. Potter, S. Kurinec, W. Park, J. Penczek, B. K. Wagner, and C. J. Summers, J. Appl. Phys. 84, 4466 (1998).

${ }^{18}$ G. Blasse, Structure and Bonding (Springer, Heidelberg, 1980), Vol. 42, pp. 1-42.

${ }^{19}$ X. Meng, H. Akamatsu, S. Murai, K. Fujita, and K. Tanaka (unpublished). 